\title{
Editorial
}

\section{Use of low quality in Vitro Diagnostics in resource poor countries: who will bell the cat?}

\author{
Tilovatul Khondaker ${ }^{1}$, Nazia Hasan Khan ${ }^{1}$, Arifa Akram ${ }^{1}$, Nusrat Mannan ${ }^{1}$, Saif Ullah Munshi ${ }^{1}$ \\ ${ }^{1}$ Department Virology, Bangabandhu Sheikh Mujib Medical University (BSMMU) Dhaka, Bangladesh
}

Key words: In vitro diagnostics, Low quality, Resource poor countries.

By accurate detection of disease, medical diagnostics have shown huge potential for saving hundreds of thousands of lives. The "in Vitro Diagnostics (IVD) medical devices' has revolutionized the modern medical diagnostics. It ensures a patient the accurate diagnosis and rapid treatment. The IVD medical device' means a device, whether used alone or in combination, marketed by the manufacturers` for the in-vitro examination of specimens derived from the human body solely or principally to provide information of diagnosis, screening, monitoring, predisposition, prognosis, prediction, and determination physiological status. These IVDs include reagents, calibrators, control materials, specimen receptacles, software, and related instruments or apparatus or other articles. IVDs vary in complexity and cost, starting from a simple Immuno-chromatographic strip to multi-million dollar automated instruments, which can perform thousands of tests within an hour in a laboratory. Comparing with the earlier times, the use of IVD medical devices has increased many folds. Currently more than 8000 generic medical device groups are available.

World Health Organization (WHO) recommends, promotes and facilitates access to safe, reliable, appropriate and quality IVD technologies and laboratory services in an equitable manner. Manufacturing of quality products helps physicians to make a proper diagnosis and management of patients. To ensure this, the health care providers should purchase the diagnostic test kits that have sufficient sensitivity, specificity, positive and negative predictive values. There are various quality guidelines including Good Manufacturing Practice (GMP), Good Laboratory Practice (GLP), Good Clinical Practice (GCP) etc.

$\triangle \square$ Correspondence:

Professor Dr. Saif Ullah Munshi

Department of Virology, BSMMU, Dhaka

Tel: 01711376343

Email: saifmunshi@gmail.com which have been established by International Conference of Harmonization (ICH) and have been adopted by USA, EU and others countries. Good Manufacturing Practice (GMP) is a system for ensuring that products are consistently produced and controlled according to quality standards. GCP describes the quality requirements for clinical trials; GLP describes quality requirements for laboratories and research organizations to ensure consistency and reliability of results. The International Organization for Standardization (ISO) has developed a standard for quality management system for medical devices called ISO 13485. This standard is based on ISO 9001:2000 and helps companies to implement and maintain a quality management system. These standards are recognized by many countries as a way to reach GMP. Several certification marks like "CE (Conformité Européenne)" or "FDA (Food and Drug Administration)" is marked on commercial IVD products. These marks indicate acceptable product standard and a claim that the manufacturer has tested the product to verify compliance with that standard set by certifying organizations. This type of declaration about the medical devices ensures the safety, efficiency and superiority of the products.

Though standards for registration of medical devices are maintained by most of the first world countries, this process is missing in developing countries. In many developed countries, guidelines are developed for pre-market evaluation, post-market surveillance, quality systems, auditing and clinical safety/performance by the authorized organizations. In those countries, several accreditation bodies are established to regulate both the private and public institutions for ensuring the standard service delivery, product quality as well as for detecting the deficiencies that are hindering the improvement and providing opportunities to rectify for future thereby safeguarding the public. Unfortunately, even after having increasing demand of IVDs in the health sector of third world countries like Bangladesh, there is lack of such type of national regulatory processes or standard 
organizations. This deficiency in regulatory supervision is degrading the quality of the health care system. Most of the developing countries do not have technical support and sufficient resources to develop their own infrastructure that will assure quality of both imported as well as indigenously produced diagnostic reagents and kits. As a result, IVDs are often marketed in the developing world without any formal evaluation of their performance and effectiveness. In the absence of any policy guidelines and adequate infrastructure, the health laboratories offers substandard diagnostic kits, thus adversely affecting the quality of clinical care and public health activities. This situation demands a more comprehensive approach for regulation and enforcement of law against substandard IVDs in resource-poor settings. For the safety and effectiveness of drugs, although a national regulatory process is established in developing countries, which has done much to improve the standardization and quality of drug, drug trials; unfortunately regulatory standards are often lacking for IVDs. In all the countries of the SouthEast Asia Region of World Health Organization (WHO SEARO), a national regulatory authority for assuring quality of pharmaceutical products is functional. The indigenous production and import of various pharmaceutical products is regulated by this agency with appropriate legislative support and legal framework. It is suggested that this existing legal framework could be used for the establishment of policy guidelines for quality assurance of IVDs. These guidelines may be used to structure a regulatory system and to ensure accurate diseases diagnosis in these countries. It is hoped that a comprehensive approach would help to increase access of good quality of medical diagnostic kits to the patients. The problem that happened in Aesop's famous fable was the technical complexity for mice to determine precisely how to solve their "cat trouble" by placing a bell around its neck. Similarly the present problem of use of non-standardized and low quality IVDs for medical diagnosis is well understood but how and who will stop this is the crux of matter.

\section{Further reading:}

1. Global Regulatory Requirements for Medical Devices, Sandra Brolin

2. Global Harmonization Task Force, http://www.ghtf.org, $11 / 4 / 2008$

3. http://www.fda.gov/MedicalDevices/DeviceRegulationand Guidance/IVDRegulatoryAssistance/ucm123682.htm\#1, accessed on 28 March 2016.

4. European Commission, Directive 93/42/EEC, published $14 / 06 / 1993$ and valid to $31 / 12 / 2008$ at http://eurlex.europa.eu

5. Guidelines on Quality of Diagnostic Reagents for Health Laboratories, World Health Organization Regional Office for South-East Asia, New Delhi, December 2001

6. Mabey, D., Peeling, R.W., Ustianowski, A. \& Perkins,M. Diagnostics for the developing world. Nature Rev.Microbiol. 2004; 2: 231-240

7. Bossuyt, P. M. et al. Towards complete and accurate reporting of studies of diagnostic accuracy: the STARD Initiative. Clin. Chem. 2003; 49: 1-6 